\title{
BMJ Open Parallel and serial mediation analysis between pain, anxiety, depression, fatigue and nausea, vomiting and retching within a randomised controlled trial in patients with breast and prostate cancer
}

\author{
Andreas Charalambous, ${ }^{1,5,2}$ Margarita Giannakopoulou, ${ }^{2}$ Evaggelos Bozas, ${ }^{2}$ \\ Lefkios Paikousis ${ }^{3}$
}

To cite: Charalambous A, Giannakopoulou M, Bozas E, et al. Parallel and serial mediation analysis between pain, anxiety, depression, fatigue and nausea, vomiting and retching within a randomised controlled trial in patients with breast and prostate cancer. BMJ Open 2019:9:e026809. doi:10.1136/ bmjopen-2018-026809

- Prepublication history for this paper is available online. To view these files, please visit the journal online (http://dx.doi org/10.1136/bmjopen-2018026809).

Received 20 September 2018 Revised 30 0ctober 2018 Accepted 28 November 2018

Check for updates

(C) Author(s) (or their employer(s)) 2019. Re-use permitted under CC BY-NC. No commercial re-use. See rights and permissions. Published by BMJ.

${ }^{1}$ Cyprus University of Technology, Limassol, Cyprus ${ }^{2}$ University of Athens, Athens, Greece

${ }^{3}$ Statistics, Improvast, Nicosia, Cyprus

${ }^{5}$ Nursing Science, University of Turku, Turku, Finland

Correspondence to Dr Andreas Charalambous; andreas.charalambous@cut. ac.cy

\section{ABSTRACT}

Objective Cancer treatment is a particularly stressful period for the patient. The reasons vary and include fear of treatment outcome as well as treatment induced side effects. The patient frequently experiences simultaneously various side effects resulting in a diminishing of the patient's health-related quality of life (HRQLL). The study provides evidence on the co-occurrence and inter-relations between pain, anxiety, depression and fatigue in patients with breast and prostate cancer.

Design This paper presents a secondary analysis of the data from a randomised control trial designed to test the effectiveness of guided imagery and progressive muscle relaxation on pain, fatigue, anxiety and depression. Nonparametric bootstrapping analyses were used to test the mediational model of anxiety, fatigue and depression as parallel mediators of the relationship between pain and HRQDL.

Setting The study was undertaken at the home setting. Participants In total 208 patients were included in the study (assigned equally in two groups), referred at the outpatient clinics of the three participating cancer care centres.

Results The three mediators fully mediate the relationship between pain and $\mathrm{HRQOL}$ indirect effect $\left(\mathrm{IE}_{\text {overall }}=-0.3839\right.$, 95\% Cl: lower limit $(\mathrm{LL})=-0.5073$ to upper limit $(U L)=-0.2825)$ indicating that patients with increased pain are likely to have higher levels of anxiety, fatigue and depression. Gender significantly moderated the mediational effect of Fatigue Index of Moderated Mediation $(\mathrm{IMM}=-0.2867 \mathrm{SE}=0.1526, \mathrm{LL}=-0.6127, \mathrm{UL}=-0.0226)$ but did not moderate mediational effect of anxiety $(\mathrm{IMM}=-0.0709, \mathrm{SE}=0.1414, \mathrm{LL}=-0.3459, \mathrm{UL}=+0.2089)$. The results show that the three mediators in a serial causal order fully mediate the relationship between pain and $\mathrm{HRQOL}\left(\mathrm{IE}_{\text {overall }}=-0.384,95 \% \mathrm{Cl}: \mathrm{LL}=-0.51\right.$ to $U L=-0.284$ ) and the ratio of the overall indirect effect to the total effect is $0.8315(95 \% \mathrm{Cl}: \mathrm{LL}=0.5683$ to $\mathrm{UL}=1.1718$ ).

Conclusion This work provides evidence that targeting fatigue, anxiety and depression may have a meaningful
Strengths and limitations of this study

- Understanding the mediating effects of symptoms within a cluster will explain how these are manifested in clinical practice.

- The findings will have important clinical implications and could guide symptom cluster management strategies.

- The study provided evidence that as part of a management strategy, by targeting fatigue, anxiety and depression may have a meaningful effect on pain.

- Rigorous design and implementation allow for the generalisability of the findings in these group of patients.

- The unavailability of longitudinal data limits the support of a definite model, which would demonstrate if the correlations between symptoms found in this study are stable over time.

effect on pain as a related symptom and potentially have a positive impact on HRQoL of patients with breast and prostate cancer

Trial registration number NCT01275872; Post-results.

\section{INTRODUCTION}

Cancer treatments and cancer itself are a source of many symptoms and side effects ${ }^{12}$ It is reported that on average patients with cancer experience 11-13 concurrent symptoms, while for patients with advanced disease the number can be even greater. ${ }^{3}$ Often these symptoms are related to each other, called symptom clusters (SCs) or symptom constellations. ${ }^{3}$ Dodd et $a t^{-6}$ defined symptom cluster as three concurrent and related symptoms that may or may not have a common aetiology. However, the authors suggested that a cluster can be composed of just two or 
more symptoms that form a stable group. ${ }^{3}$ Symptoms can be related through a common mechanism or aetiology, by sharing common variance, or by producing different outcomes than individual symptoms.

The impact of these clusters can be accumulative and debilitating for the patient and far more serious and persistent than single symptoms. Their concurrent presence makes their clinical management complex and challenging. As a result, these clusters can affect the patient's overall health-related quality of life (HRQoL) ${ }^{7}$ and significantly affect the patient's sense of well-being and his or her physical and social functions. ${ }^{8}$

A number of studies have tried to record the most common clusters in various cancer types, ${ }^{49}$ however, due to the lack of agreement about a robust, clinically relevant definition of SCs, their results should be interpreted with caution. Although the groups of symptoms that tended to cluster were identified, there is limited consistency in the way in which these SCs and their associated variables were identified. ${ }^{1}$

Preceding studies, again by drawing on the weaknesses of the clinical definition of an SC, they provide limited information towards understanding the way (or ways) the symptoms are actually correlated to each other or to other variables (ie, HRQoL). Most of the models available adopt a serial mediation approach to the study of these clusters, suggesting a rather linear correlation between the symptoms.

According to the definition by Dodd $e t a l,{ }^{4}$ for example, a cluster of pain, fatigue and insomnia is presented as a legitimate one. However, the authors supported that pain leads to fatigue and in turn insomnia and this is a rather linear representation. Therefore, the rationale for examining these symptom clusters within the mediational models is to explicate whether these symptoms can have an alternative way of interaction other than the one suggested in a linear way. What is further lacking in the current research is the role of other possible mediators such as cancer diagnoses which is also explored within this study.

These limitations are present with other SCs that have been identified so far in the literature leaving a gap to the best understanding of this phenomenon but most importantly to the way it can be best clinically managed. When interventions are directed to ameliorate a particular symptom within a cluster, other symptoms within the cluster may be relieved. However this evidence is not available to the best of our knowledge in the suggested mediational models available in the literature. As a result, current practice is driven based on a priori assumptions about the relationships among symptoms resulting in targeting the 'dominant' symptom. In the previous example, pain would have been identified as a 'dominant' symptom, and therefore, secondary attention is attributed to any other symptom that co-occurs.

These a priori assumptions are however not based on scientific evidence but rather have been established on clinical experience. However, a symptom cluster experienced by the patient creates a complex condition where pain is rarely reported as a single symptom, but as a distressing symptom is reported and addressed in a timely manner. However, the lack of knowledge in the ways symptoms might correlate to each other and with other variables, the question remains whether the dominant symptom in these situations is indeed pain. The question that also arises is whether there would be a clinical benefit if, for example, pain and fatigue are correlated and interventions were specifically designed to target both symptoms. The researchers so far had hypothesised that it is pain and this has become the common practice dominating the current clinical management of this specific SC. This study hypothesises that in the mediational models to be tested within this cluster of symptoms, fatigue, anxiety and depression will be identified as mediating factors in the pain-HRQoL linkage. Furthermore, the study hypothesises that the mediation role of anxiety, depression and fatigue will differ between the breast cancer and prostate cancer diagnoses.

\section{Aim}

The aim of this study was to provide evidence on the co-occurrence of and inter-relations between symptoms occurring as part of a cluster in two groups of patients diagnosed with breast and prostate cancer.

\section{METHODS}

The data for the purpose of this paper came from a randomised control trial (NCT01275872) designed to test the effectiveness of guided imagery (GI) and progressive muscle relaxation (PMR) on a cluster of symptoms reported by patients diagnosed with breast or prostate cancer when receiving chemotherapy. ${ }^{10}$ The patients in the study reported the following symptoms: fatigue, pain, nausea, vomiting and retching, anxiety and depression.

\section{Measurements}

The data were collected through the use of appropriate valid and reliable measures. The pain intensity was assess through a 10-point Numeric Rating Scale (NRS) for pain where 0 indicated the absence of pain and 10 indicated the worst experienced level of pain. Within the cancer context, NRS used to assess the intensity of pain have a proven validity and reliability. ${ }^{11}$

The Cancer Fatigue Scale $(\mathrm{CFS})^{12}$ was used to assess the participants' levels of fatigue. The CFS consists of 3 dimensions with 15 items that assess patients' responses on physical, affective and cognitive aspects of their daily living. A 5-point Likert scale is used to assess each of the items where 1 denotes 'not at all' to 5 which denotes 'very much'. The possible scores range from 0 to 28 for the physical, 0 to 16 for the affective and 0 to 16 for the cognitive subscale. The CFS had good stability (average test-retest reliability $\mathrm{r}=0.69, \mathrm{p}<0.001$ ) and good internal consistency (Cronbach's alpha coefficient for all 15 items $=0.88) .{ }^{13}$ 
Nausea and vomiting were assessed through the Revised Rhodes Index of Nausea, Vomiting and Retching $(\mathrm{INVR})^{14}$ which consists of eight 5-point self-reported items designed to assess subjective and objective factors of nausea, vomiting and retching. The validity and reliability of the INVR in patients with cancer have been demonstrated in preceding studies. ${ }^{15}$

The levels of anxiety were assessed with the Zung SelfRating Anxiety Scale which contains 20 items that assess physiological and psychological symptoms commonly associated with anxiety, and each item is answered on a 4-point Likert-type scale ranging from 'never' to 'always'. ${ }^{16}$ Raw scores sum to $20-44$ that signify normal anxiety levels, 45-59 signifying mild to moderate anxiety levels, 60-74 signifying moderate to severe anxiety levels and finally 75-80 indicating extreme anxiety levels. The scale's validity and reliability in cancer populations have been established in previous studies. ${ }^{17}$

The Beck Depression Inventory-II has been used to assess patients' level of depression according to 21 items that correspond to a specific symptom common among people with depression. ${ }^{18}$ Each item is evaluated on a 4 -point scale ranging from 0 to 3 . The number of items' responses is summed to indicate the severity of depression as follows: 1-10 is considered normal, 11-16 indicated mild mood disturbance, 17-20 indicates borderline clinical depression, 21-30 represents moderate depression, 31-40 represents severe depression and over 40 indicates extreme depression. The Beck Depression Inventory has been extensively used in cancer populations where it demonstrated excellent validity and reliability. ${ }^{19}$

As with the majority of studies that explore symptom and symptom management, QoL is a frequently reported outcome that has been used to demonstrate the negative impact of symptoms but also to reflect on the effectiveness of symptom management strategies. HRQoL was included in the study due to the consistent evidence in the literature that the cluster of symptoms is associated to poor levels of QoL in patients diagnosed with cancer. $^{72021}$ Therefore, HRQoL has also being introduced in this study as a variable that can be correlated to the studied symptoms. The HRQoL of the patients was assessed with the EORTC QLQ-C30- European Organization for Research and Treatment of Cancer Quality of Life Questionnaire in addition to the module BR23 for patients with breast cancer and the module PR25 for patients with prostate cancer. The EORTC QLQ-C30 includes five functional scales (physical, role, cognitive, emotional and social), three symptom scales (fatigue, pain, nausea and vomiting), a global health status/ QoL scale, and a number of single items assessing additional symptoms (dyspnoea, loss of appetite, insomnia, constipation and diarrhoea) and perceived financial impact of the disease. The research items are assessed on a Likert scale ranging from 1 to 4 ( $1=$ "'not at all', $2=$ 'a little', $3=$ ='quite a bit', $4=$ "very much'). Only the last two items assessing overall health and overall QoL are assessed on a scale ranging from 1 (very poor) to 7 (excellent). ${ }^{22}$
The EORTC QLQ-C30 module has a proven record of excellent validity and reliability across different cancer populations and languages. ${ }^{23}$

The BR23 is a breast-specific module that comprises 23 questions to assess body image, sexual functioning, sexual enjoyment, future perspective, systemic therapy side effects, breast symptoms, arm symptoms and upset by hair loss. The questions of the module are assessed on a Likert scale ranging from 1 to 4 (1='not at all'; 2='a little'; $3=$ 'quite a bit'; $4=$ =very much'). The validity and reliability of the BR23 module have been demonstrated in breast cancer populations. ${ }^{24}$

The PR 25 prostate-specific module consists of 25 items across 6 scales: urinary symptoms, incontinence aid, bowel symptoms, hormonal treatment-related symptoms, sexual active and sexual function. The module includes five conditional questions, conditioned on the need of incontinence aid and the status of being sexually active. The questions of the module are assessed on a Likert scale ranging from 1 to 4 (1='not at all'; 2='a little'; $3=$ ='quite a bit'; $4=$ ='very much'). The validity and reliability of the PR25 module have been extensively demonstrated in prostate cancer populations. ${ }^{25}$

\section{Sample}

As the rationale for this study was to test the correlations between symptoms identified as clusters, only the baseline (T0) measurements from both groups (intervention and control group) were included in the analysis. Based on predetermined criteria, 208 patients were included in the study. The sample included 104 female patients with breast cancer (52 in control group +52 in intervention group) and 104 patients with prostate cancer (52 in control +52 in intervention).

\section{Statistical analysis}

Non-parametric bootstrapping analyses ${ }^{26}$ were deployed to test the mediational model of anxiety, fatigue and depression as parallel mediators of the relationship between pain and HRQoL. The nausea, vomiting and retching were excluded from the analyses as these were not found to correlate with the other symptoms. In these analyses, mediation is significant if the $95 \%$ bias corrected and accelerated CIs (lower limit, LL), upper limit (UL) for the indirect effect (IE) do not include $0 .{ }^{826}$ Moreover, separated regression analyses were deployed to explore the statistical association of cancer diagnosis and age of the patient with the HRQoL.

Three mediation models are hypothesised and explored. The first model is a cancer diagnosis adjusted parallel mediation model of IE of pain to HRQoL through anxiety, fatigue and depression. The second model is a moderated mediation model where the IEs of the mediating factors are explored on each cancer diagnosis (conditional IEs). The third model assumes a serial causal chain of the three mediators (serial mediation). Six different causal chains were explored in this model. 
All involved variables in the analyses were standardised (z scores) before running the analyses, hence standardised coefficients are reported for the total, direct and IEs.

Analysis was performed by using the PROCESS function V.2.16.1 in SPSS V.21. The model 4 (model as a parameter in the PROCESS function) was used for the parallel mediation model, model 15 (moderated mediation) for the second mediation model and model 6 for the serial mediation models (SMMs). ${ }^{27}$

Model fit is also reported using the following: a $\chi^{2}$ (df, a Comparative Fit Index (CFI) $>0.90$ and a root mean square error of approximation of $<0.10$. The fit indices were derived using the package lavaan in $\mathrm{R}^{28}$

A detail presentation of the study's methodology is presented elsewhere. ${ }^{29}$

\section{Patient and public involvement statement}

As part of the study, a group of patients with relevant diagnoses were consulted in identifying the study's research question and relevant hypotheses. The patients were not directly involved in the design of the study nor in the recruitment and carrying out of the study. The authors intend to disseminate results to study participants through written summaries; academic outputs will be publicised through traditional media channels and social media.

\section{RESULTS}

\section{Sample characteristics}

The sample consisted of 104 male (52 in the intervention group and 52 in the control group) and 104 female (52 in the intervention group and 52 in the control group) patients diagnosed with prostate and breast cancer. Eighty-six patients with prostate cancer were diagnosed with stage T3a, Gleason score 8 and the remaining 18 with stage T3b, Gleason score 9. Patients with breast cancer were all diagnosed with clinical stage T3N1M0. Most of the participants belong to the $41-50$ and the 51-60 age groups $(38.9 \%$ and $26.4 \%$, respectively). Patients with prostate cancer were treated either with a combination of androgen deprivation therapy (ADT) and adjuvant chemotherapy or with a combination of ADT and radiation $(65.3 \%$ and $19.2 \%$, respectively).

The correlation analysis shows that HRQoL is negatively associated with pain $(\mathrm{r}=-0.462)$, fatigue $(\mathrm{r}=-0.601)$, anxiety $(\mathrm{r}=-0.595)$ and depression $(\mathrm{r}=-0.510)$ indicating that lower QoL is associated with higher levels of the psychological aspects as well as pain. Furthermore, pain is positively correlated with all psychological aspects and negatively correlated with HRQoL (table 1).

\section{Covariates}

Age was not found to be statistically associated with the level of HRQoL either on the total effects model ( $b=-1.13$ $\mathrm{p}=0.27$, predictors of HRQoL: pain, sex, age) or on the direct effect model $(b=-0.9903 \mathrm{p}=0.2866$, predictors to HRQoL: anxiety, fatigue, depression, pain, gender, age).

Gender was found to be statistically associated with the HRQoL in the total effects model $(b=-7.2972 p=0.0143)$ indicating that female patients experienced lower QoL compared with male patients. Therefore, gender, which in this study also reflects the type of cancer, is included in the mediation models as a statistical control variable.

\section{Parallel mediation model}

Results based on 5000 bootstrapped samples indicated that, controlling for the gender, while the total effect of pain on HRQoL was significant $\left(\beta_{\text {total }}=-0.4616, \mathrm{SE}=0.0612\right.$, $\mathrm{p}<0.001)$, the direct effect was not $\left(\beta_{\text {direct }}=-0.0778\right.$, $\mathrm{SE}=0.0685, \mathrm{p}=0.2576$ ) and IEs are present (figure 1 ).

Overall, the three mediators fully mediate the relationship between pain and HRQoL ( $\mathrm{IE}_{\text {overall }}=-0.3839,95 \%$ CI: $\mathrm{LL}=-0.5073$ to $\mathrm{UL}=-0.2825$ ) indicating that patients with increased pain are more likely to have higher levels of anxiety, fatigue and depression. Patients through the experience of high levels of anxiety, fatigue and depression are more likely to report lower levels of QoL.

Two out of the three mediators were found to significantly contribute to the overall IE. Specifically, there is as statistically significant IE of pain to HRQoL though anxiety $\left(\mathrm{IE}_{\text {anxiety }}=-0.1378,95 \%\right.$ CI: $\mathrm{LL}=-0.2615$ to $\left.\mathrm{UL}=-0.0395\right)$, such that participants who indicated high levels of pain were more likely to feel anxiety, and through high levels of anxiety, more likely to report lower levels of HRQoL. In addition, there is as statistically significant IE of pain to HRQoL though fatigue ( $\mathrm{IE}_{\text {fatigue }}=-0.1856,95 \% \mathrm{CI}$ : $\mathrm{LL}=-0.2716$ to $\mathrm{UL}=-0.1093$ ), such that patients who indicated high levels of pain were more likely to feel fatigue, and through high levels of fatigue, more likely to report reduced HRQoL. Depression does not mediate the relationship between pain and HRQoL ( $\mathrm{IE}_{\text {depression }}=-0.0605$, 95\% CI: $\mathrm{LL}=-0.1575$ to $\mathrm{UL}=0.0289$ ).

\begin{tabular}{|c|c|c|c|c|c|}
\hline & Pain & Fatigue & Anxiety & Depression & HRQoL \\
\hline Pain & 1 & & & & \\
\hline Fatigue & $0.567^{\star \star}$ & 1 & & & \\
\hline Anxiety & $0.590^{* *}$ & $0.715^{\star \star}$ & 1 & & \\
\hline Depression & $0.541^{\star \star}$ & $0.565^{\star \star}$ & $0.735^{\star *}$ & 1 & \\
\hline HRQoL & $-0.462^{\star *}$ & $-0.601^{\star \star}$ & $-0.595^{\star \star}$ & $-0.510^{\star \star}$ & 1 \\
\hline
\end{tabular}

${ }^{\star *} \mathrm{P}<0.001$.

$\mathrm{HRQ}$ L, health-related quality of life. 


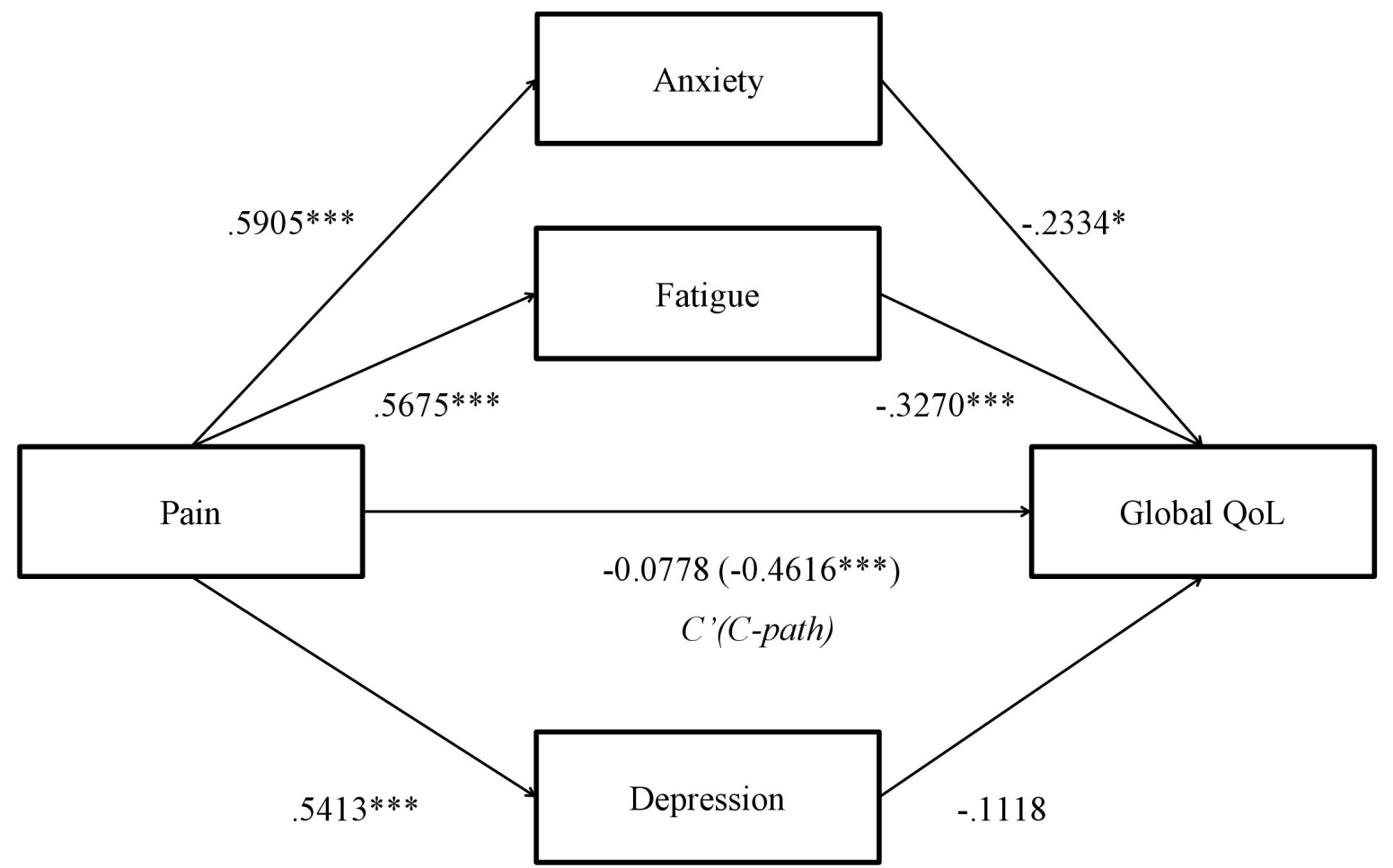

Figure 1 Parallel mediation model ( $n=208)$. Indirect effects of pain on Global HRQoL through anxiety, fatigue and depression. Model is controlled for the cancer diagnosis. Standardised effects are presented. The effects on the direct path from pain to global HRQoL depict the direct effect and the (total effect). ${ }^{*} \mathrm{P}<0.05$, ${ }^{* * *} \mathrm{P}<0.001$. HRQoL, health-related quality of life; QoL, quality of life.

Specific IE contrasts between the proposed mediators do not show statistically significant difference between the IEs of anxiety and fatigue ( $b=0.0478,95 \% \mathrm{CI}$ : $\mathrm{LL}=-0.260$ to $\mathrm{UL}=0.0822)$. The ratio of the overall IE to the total effect is 0.8315 ( $95 \%$ CI: $\mathrm{LL}=0.5683$ to $\mathrm{UL}=1.1718$ ), while the ratio of the anxiety and fatigue IEs to the total effect is 0.2985 (95\% CI: $\mathrm{LL}=0.0758$ to $\mathrm{UL}=0.5711$ ) and 0.4020 ( $\mathrm{LL}=0.2230, \mathrm{UL}=0.6317)$, respectively.

\section{Moderated mediation model: cancer diagnosis moderation}

The current analysis tests whether the IE of pain to HRQoL through anxiety or fatigue is moderated by cancer diagnosis, that is, whether the mediation effect observed earlier is statistically significantly different in patients with prostate and breast cancer.

A moderated mediation model is explored where anxiety and fatigue act as parallel mediators and diagnosis as a moderator (figure 2) to the mediation. The proposed model is under model 15 of the PROCESS documentation $^{27}$ where the moderation effect takes place at the B-path (mediator to dependent) and at the C-path (direct path: independent to dependent). Model 15 (moderation effect not included to the A-path), was chosen over model 59 (moderation effect on all paths) since separate regression analysis for the interaction of diagnosis to the relationship of the independent (pain) to the mediators (anxiety and fatigue) did not reach statistical significance. The slope of the line relating the IE to the moderator is the 'Index of Moderated Mediation' (IMM) ${ }^{28}$ The statistical significance of the IMM effect is assessed along with the conditional IEs across each cancer diagnosis.

Diagnosis significantly moderated the mediational effect of fatigue (IMM=-0.2867 $\mathrm{SE}=0.1526$, $\mathrm{LL}=-0.6127$, $\mathrm{UL}=-0.0226$ ) but did not moderate mediational effect of anxiety $(\mathrm{IMM}=-0.0709, \quad \mathrm{SE}=0.1414, \quad \mathrm{LL}=-0.3459$, $\mathrm{UL}=+0.2089$ ). This means that there is a meaningful difference in the magnitude of the conditional IEs of each cancer diagnosis in the mediation effect of pain to HRQoL through anxiety. Specifically, we observe that, for patients with prostate cancer, the conditional IE is trivial and not statistically significant $(\beta=0.0746, \mathrm{SE}=0.1474, \mathrm{LL}=-0.1893, \mathrm{UL}=+0.3853$ ) but for patients with breast cancer, the conditional IE is stronger than prostate cancers' and statistically significant $(\beta=-0.2121, \mathrm{SE}=0.0434, \mathrm{LL}=-0.307, \mathrm{UL}=-0.1351)$ (table 2$)$. Fatigue is a significant mediator to the relationship between pain and HRQoL in patients with breast cancer rather than patients with prostate cancer. There is no meaningful difference in the mediating effect of anxiety for the relationship between pain and HRQoL between prostate and breast cancer diagnoses.

\section{Serial mediation model}

Serial mediation hypothesises a causal chain linking of the mediators (anxiety, fatigue, depression), with a specified direction flow. For example, pain could increase anxiety, which in turn increases depression which could in turn increase fatigue and thus decrease QoL (ie, Pain->Anxiety->Depression-Fatigue->HRQoL). 


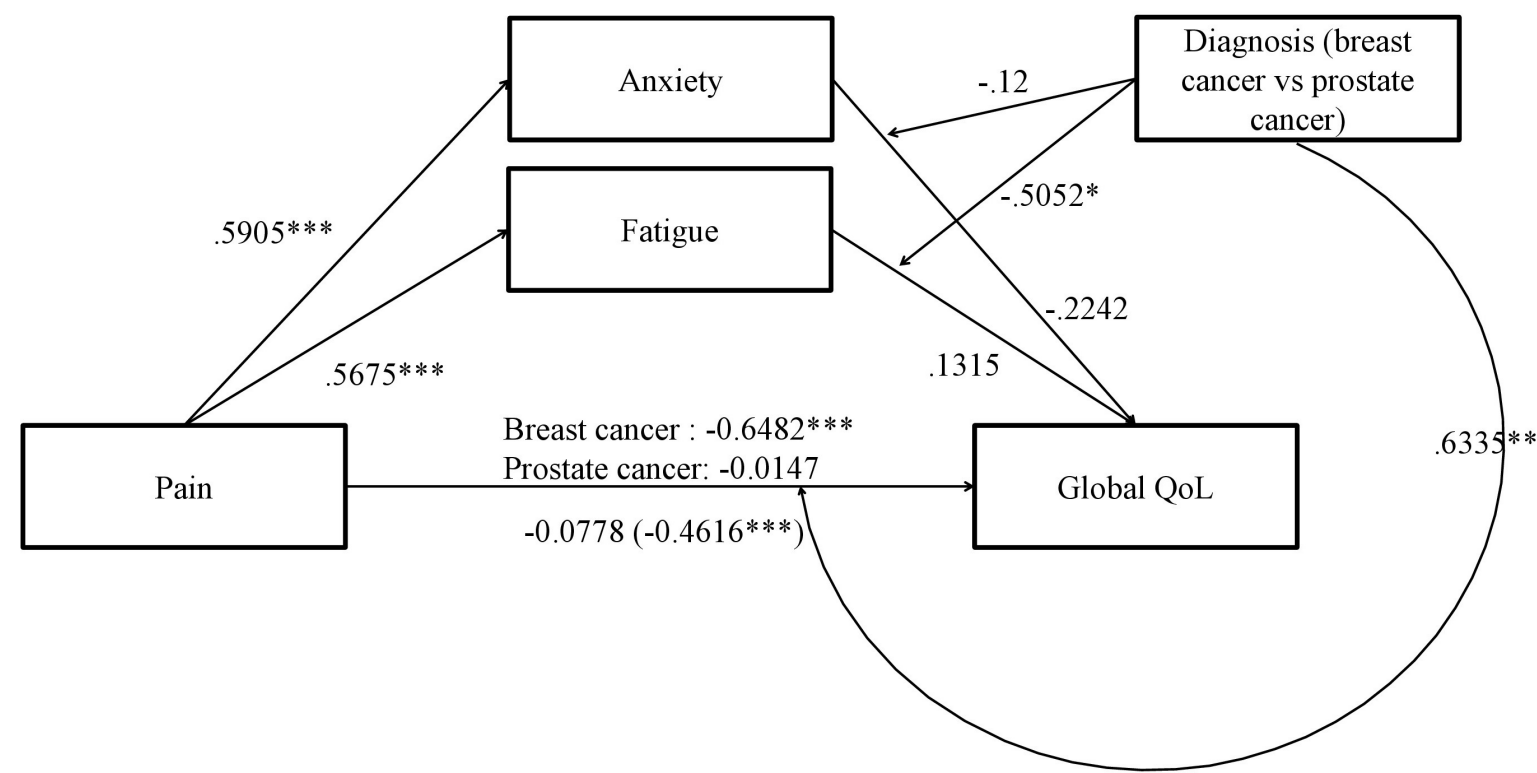

Figure 2 Moderated mediation model $(\mathrm{n}=208)$. Conditional indirect effects on cancer diagnosis (breast cancer coded as 1 and prostate cancer as 0 ) of pain on global HRQoL through depression. Note: Standardised effects are presented. The effects on the direct path from pain to global HRQoL depict the conditional direct effects for each cancer diagnosis as well as the unconditional direct effect C' path (total effect C-path). The effects of the moderator diagnosis to the paths represent the interaction slopes. Effects on the B-paths from the mediators to HRQoL represent the simple slopes. ${ }^{\star} \mathrm{P}<0.05,{ }^{* *} \mathrm{P}<0.01$, ${ }^{\star \star *} P<0.001$. HRQoL, health-related quality of life; QoL, quality of life.

The results show that the three mediators in a serial causal order (any order that is) fully mediate the relationship between pain and HRQoL ( $\mathrm{IE}_{\text {overall }}=-0.384,95 \%$ CI: $\mathrm{LL}=-0.51$ to $\mathrm{UL}=-0.284$ ) and the ratio of the overall IE to the total effect is 0.8315 (95\% CI: $\mathrm{LL}=0.5683$ to $\mathrm{UL}=1.1718$ ). The total IE and the ratio to the total effect, both are the same as in the parallel mediation model explored earlier.

Since three mediators were used, six different causal order models were produced (table 3 ). All six models were compared in terms of the significant path created by each different causal order of the mediators. SMM 1, SMM 2 and SMM 4 yielded only three significant indirect paths out of the seven possible paths, whereas SMM 3, SMM 5 and SMM 6 yielded 4, 5 and 6 significant paths, respectively.

SMM 3, SMM 5 and SMM 6 yielded a significant indirect path involving all three mediators in a causal chain. The path Pain->Depression->Anxiety->Fatigue- $>$ HRQoL in SMM 3 yielded the highest ratio of indirect to total effect; 0.126 (95\% CI 0.056 to 0.248 ) among the three models (table 3 ).

The indirect paths involving fatigue and depression (one after the other and vice versa) were statistically significant in one out of the six SMMs and specifically in SMM6.

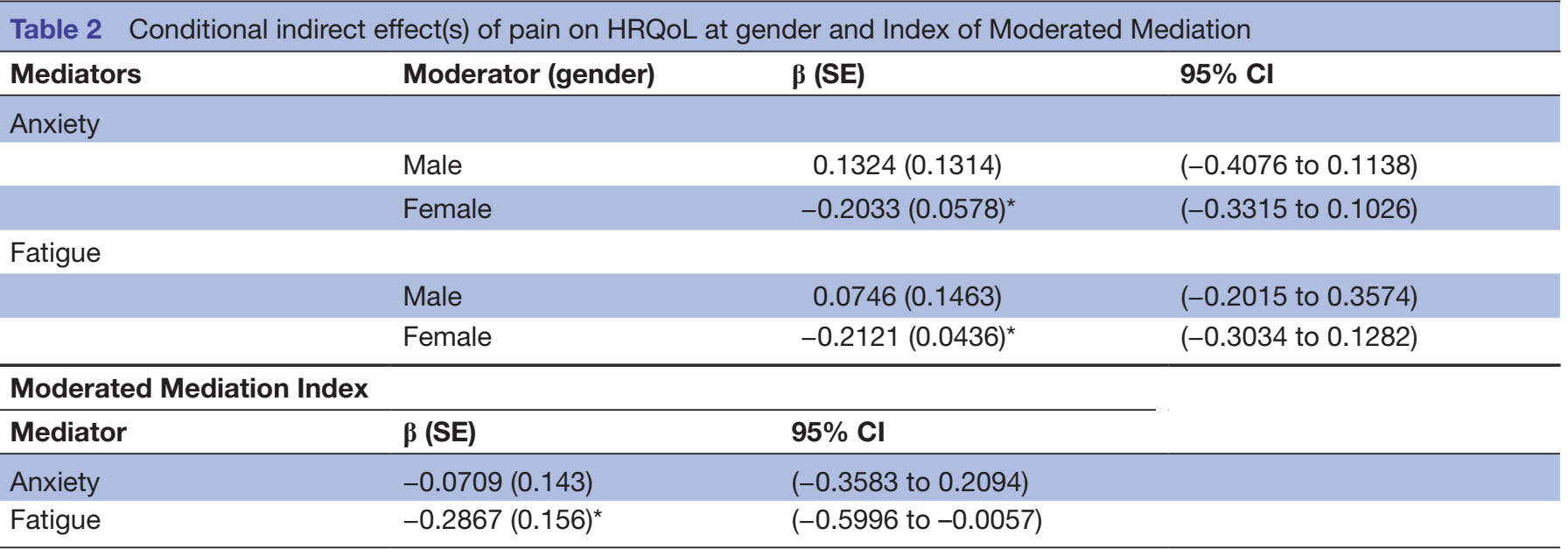

Bias-corrected bootstrapped Cls, 5000 bootstrap samples. Statistically significant moderated mediation occurs only for fatigue. Although there is a significant conditional indirect effect for females through the mediator anxiety, the difference between the conditional indirect effect for males is not significantly different than that of the females.

${ }^{*} \mathrm{P}<0.05$. 
Table 3 Standardised indirect effects and ratio of indirect to total effects for the paths on the SMMs

\begin{tabular}{|c|c|c|c|c|}
\hline & \multicolumn{2}{|c|}{ Indirect effects of pain on HRQoL } & \multicolumn{2}{|c|}{ Ratio of indirect to total effect } \\
\hline & b (boot SE) & BC 95\% Cl (LL-UL) & b (boot SE) & BC 95\% CI (LL-UL)* \\
\hline \multicolumn{5}{|c|}{ SMM1: PN ->AXT ->DEP ->FTG ->HRQoL } \\
\hline PN ->AXT ->HRQoL & $-0.138(0.057)$ & $(-0.268$ to -0.04$)$ & $0.299(0.126)$ & (0.082 to 0581$)$ \\
\hline $\mathrm{PN}->\mathrm{AXT}->\mathrm{DEP}->\mathrm{HRQoL}$ & $-0.042(0.033)$ & $(-0.109$ to 0.022$)$ & $0.091(0.074)$ & $(-0.049$ to 0.239$)$ \\
\hline PN ->AXT ->FTG ->HRQoL & $-0.108(0.032)$ & $(-0.186$ to -0.057$)$ & $0.234(0.077)$ & (0.114 to 0.424$)$ \\
\hline $\begin{array}{l}\text { PN }->\text { AXT ->DEP }->\text { FTG } \\
->\text { HRQoL }\end{array}$ & $-0.004(0.01)$ & $(-0.028$ to 0.013$)$ & $0.009(0.022)$ & $(-0.029$ to 0.063$)$ \\
\hline PN ->DEP ->HRQoL & $-0.018(0.018)$ & $(-0.069$ to 0.005$)$ & $0.04(0.04)$ & $(-0.012$ to 0.153$)$ \\
\hline PN $->$ DEP $->$ FTG $->$ HRQoL & $-0.002(0.005)$ & $(-0.019$ to 0.005$)$ & $0.004(0.011)$ & $(-0.01$ to 0.041$)$ \\
\hline PN ->FTG ->HRQoL & $-0.071(0.024)$ & $(-0.13$ to -0.033$)$ & $0.154(0.056)$ & (0.07 to 0.296$)$ \\
\hline \multicolumn{5}{|c|}{ SMM2: PN ->FTG ->AXTDEP ->HRQoL } \\
\hline PN ->FTG ->HRQoL & $-0.186(0.042)$ & $(-0.272$ to -0.11$)$ & $0.299(0.126)$ & (0.082 to 0.581$)$ \\
\hline PN ->FTG ->AXT ->HRQoL & $-0.074(0.032)$ & $(-0.146$ to -0.023$)$ & $0.244(0.077)$ & (0.119 to 0.427$)$ \\
\hline PN ->FTG ->DEP ->HRQoL & $-0.002(0.006)$ & $(-0.022$ to 0.006$)$ & $0.088(0.074)$ & $(-0.047$ to 0.241$)$ \\
\hline $\begin{array}{l}\text { PN ->FTG ->AXT ->DEP } \\
->\text { HRQoL }\end{array}$ & $-0.022(0.018)$ & $(-0.063$ to 0.011$)$ & $0.003(0.008)$ & $(-0.007$ to 0.029$)$ \\
\hline PN ->AXT ->HRQoL & $-0.064(0.03)$ & $(-0.141$ to -0.018$)$ & $0.158(0.058)$ & (0.071 to 0.304$)$ \\
\hline PN $->$ AXT $->$ DEP $->$ HRQoL & $-0.019(0.017)$ & $(-0.062$ to 0.008$)$ & $0.002(0.005)$ & $(-0.004$ to 0.024$)$ \\
\hline PN ->DEP ->HRQoL & $-0.018(0.017)$ & $(-0.067$ to 0.005$)$ & $0.038(0.039)$ & $(-0.012$ to 0.147$)$ \\
\hline \multicolumn{5}{|c|}{ SMM3: PN ->DEP > AXT ->FTG->HRQoL } \\
\hline PN ->DEP ->HRQoL & $-0.061(0.048)$ & $(-0.156$ to 0.03$)$ & $0.131(0.106)$ & $(-0.072$ to 0.343$)$ \\
\hline PN ->DEP $->$ AXT $->$ HRQoL & $-0.074(0.031)$ & $(-0.15$ to -0.021$)$ & $0.161(0.07)$ & (0.044 to 0.324$)$ \\
\hline PN ->DEP ->FTG ->HRQoL & $-0.006(0.015)$ & $(-0.043$ to 0.018$)$ & $0.014(0.032)$ & $(-0.038$ to 0.094$)$ \\
\hline $\begin{array}{l}\text { PN ->DEP ->AXT ->FTG } \\
->\text { HRQoL }\end{array}$ & $-0.058(0.019)$ & $(-0.105$ to -0.028$)$ & $0.126(0.047)$ & (0.057 to 0.248$)$ \\
\hline PN ->AXT ->HRQoL & $-0.064(0.029)$ & $(-0.138$ to -0.019$)$ & $0.138(0.065)$ & $(0.042$ to 0.306$)$ \\
\hline PN ->AXT ->FTG ->HRQoL & $-0.05(0.017)$ & $(-0.094$ to -0.025$)$ & $0.108(0.038)$ & (0.053 to 0.212$)$ \\
\hline PN ->FTG ->HRQoL & $-0.071(0.024)$ & $(-0.13$ to -0.033$)$ & $0.154(0.056)$ & (0.07 to 0.296$)$ \\
\hline \multicolumn{5}{|c|}{ SMM4: PN $->$ AXT $->$ FTG $->$ DEP $>->$ HRQoL } \\
\hline PN ->AXT ->HRQoL & $-0.138(0.056)$ & $(-0.262$ to $-0,04)$ & $0.299(0.125)$ & (0.076 to 0.578$)$ \\
\hline PN ->AXT ->FTG ->HRQoL & $-0.113(0.031)$ & $(-0.187$ to -0061$)$ & $0.244(0.076)$ & (0.122 to 0.426$)$ \\
\hline PN ->AXT ->DEP ->HRQoL & $-0.041(0.034)$ & $(-0.115$ to 0.02$)$ & $0.088(0.075)$ & $(-0.045$ to 0.259$)$ \\
\hline $\begin{array}{l}\text { PN }->\text { AXT }->\text { FTG }->\text { DEP } \\
->\text { HRQoL }\end{array}$ & $-0.001(0.004)$ & $(-0.013$ to 0.003$)$ & $0.003(0.008)$ & $(-0.007$ to 0.028$)$ \\
\hline PN ->FTG ->HRQoL & $-0.073(0.025)$ & $(-0.131$ to -0.031$)$ & $0.158(0.058)$ & (0.065 to 0.299$)$ \\
\hline PN $->$ FTG $->$ DEP $->$ HRQoL & $-0.001(0.003)$ & $(-0.011$ to 0.002$)$ & $0.002(0.006)$ & $(-0.004$ to 0.022$)$ \\
\hline $\mathrm{PN}->\mathrm{DEP}->\mathrm{HRQ} \mathrm{LL}$ & $-0.018(0.017)$ & $(-0.067$ to 0.005$)$ & $0.038(0.039)$ & $(-0.01$ to 0.152$)$ \\
\hline \multicolumn{5}{|c|}{ SMM5: PN ->FTG ->DEP>AXT ->HRQoL } \\
\hline PN ->FTG ->HRQoL & $-0.186(0.042)$ & $(-0.272$ to -0.11$)$ & $0.402(0.103)$ & (0.223 to 0.632$)$ \\
\hline PN ->FTG ->DEP ->HRQoL & $-0.024(0.019)$ & $(-0.064$ to 0.011$)$ & $0.052(0.042)$ & $(-0.023$ to 0.146$)$ \\
\hline PN ->FTG ->AXT ->HRQoL & $-0.052(0.023)$ & $(-0.106$ to -0.016$)$ & $0.112(0.051)$ & (0.031 to 0.23$)$ \\
\hline $\begin{array}{l}\text { PN }->\text { FTG }->\text { DEP }->A X T \\
->\text { HRQoL }\end{array}$ & $-0.022(0.011)$ & $(-0.05$ to -0.007$)$ & $0.049(0.024)$ & (0.015 to 0.11$)$ \\
\hline PN ->DEP ->HRQoL & $-0.036(0.031)$ & $(-0.106$ to 0.016$)$ & $0.079(0.069)$ & $(-0.034$ to 0.238$)$ \\
\hline PN ->DEP ->AXT ->HRQoL & $-0.034(0.015)$ & $(-0.07$ to -0.01$)$ & $0.073(0.034)$ & (0.021 to 0.156$)$ \\
\hline PN ->AXT ->HRQoL & $-0.03(0.019)$ & $(-0.082$ to -0.004$)$ & $0.065(0.042)$ & (0.008 to 0.181$)$ \\
\hline
\end{tabular}


Table 3 Continued

\begin{tabular}{|c|c|c|c|c|}
\hline & \multicolumn{2}{|c|}{ Indirect effects of pain on HRQoL } & \multicolumn{2}{|c|}{ Ratio of indirect to total effect } \\
\hline & b (boot SE) & BC 95\% Cl (LL-UL) $)^{\star}$ & b (boot SE) & BC $95 \%$ Cl (LL-UL)* \\
\hline \multicolumn{5}{|c|}{ SMM6: PN ->DEP>FTG $->A X T$->HRQoL } \\
\hline PN ->DEP ->HRQoL & $-0.061(0.048)$ & $(-0.158$ to 0.029$)$ & $0.131(0.107)$ & $(-0.065$ to 0.355$)$ \\
\hline PN ->DEP ->FTG ->HRQoL & $-0.065(0.023)$ & $(-0.12$ to -0.03$)$ & $0.14(0.054)$ & (0.062 to 0.283$)$ \\
\hline PN ->DEP ->AXT ->HRQoL & $-0.056(0.023)$ & $(-0.111$ to -0.017$)$ & $0.122(0.053)$ & (0.033 to 0.245$)$ \\
\hline $\begin{array}{l}\text { PN ->DEP ->FTG ->AXT } \\
->\mathrm{HRQ} \text { oL }\end{array}$ & $-0.018(0.009)$ & $(-0.043$ to -0.006$)$ & $0.039(0.021)$ & (0.011 to 0.096$)$ \\
\hline PN ->FTG ->HRQoL & $-0.121(0.031)$ & $(-0.192$ to -0.07$)$ & $0.262(0.073)$ & (0.146 to 0.44$)$ \\
\hline PN $->$ FTG $->$ AXT $->$ HRQoL & $-0.034(0.016)$ & $(-0.073$ to -0.011$)$ & $0.073(0.035)$ & (0.021 to 0.16$)$ \\
\hline PN ->AXT ->HRQoL & $-0.03(0.019)$ & $(-0.082$ to -0.004$)$ & $0.065(0.042)$ & (0.008 to 0.181$)$ \\
\hline
\end{tabular}

Table shows standardised indirect effects with bootstrapped SEs; Paths in bold indicate statistically significant indirect effects.

SBias-corrected $95 \%$ Cls.

*LL- Lower Limit, UL- Upper Limit

AXT, anxiety; DEP, depression; FTG, fatigue; HRQoL, health-related quality of life; PN, pain; SMM, serial mediation model.

The indirect paths involving anxiety and depression (one after the other and vice versa) were statistically significant in three out of the six SMMs and specifically in SMM3, SMM5 and SMM6

The indirect paths involving fatigue and anxiety (one after the other and vice versa) were statistically significant in all SMMs. This result means that increased pain increases fatigue (or anxiety) which in turn increase anxiety (or fatigue) resulting in a decreased QoL. The serial causal effect of these two mediators was found significant in any casual order of the mediators in place.
Figure 3 depicts the effects of the direct paths linking pain to each mediator and among mediators resulting from the SMM 6 in which all the direct and IEs are statistically significant. The positive signs (+ve) of the effects are indicative of the increased anxiety, fatigue and depression that increased pain causes. Moreover, it shows that increased levels of each mediator is associated with a positive effect (ie, increase) in the levels of the mediator with a direct connection. All indirect paths from pain to HRQoL are negative, showing the reduction in HRQoL levels through the increase in the levels of the mediators. The SMM 6 results show that the

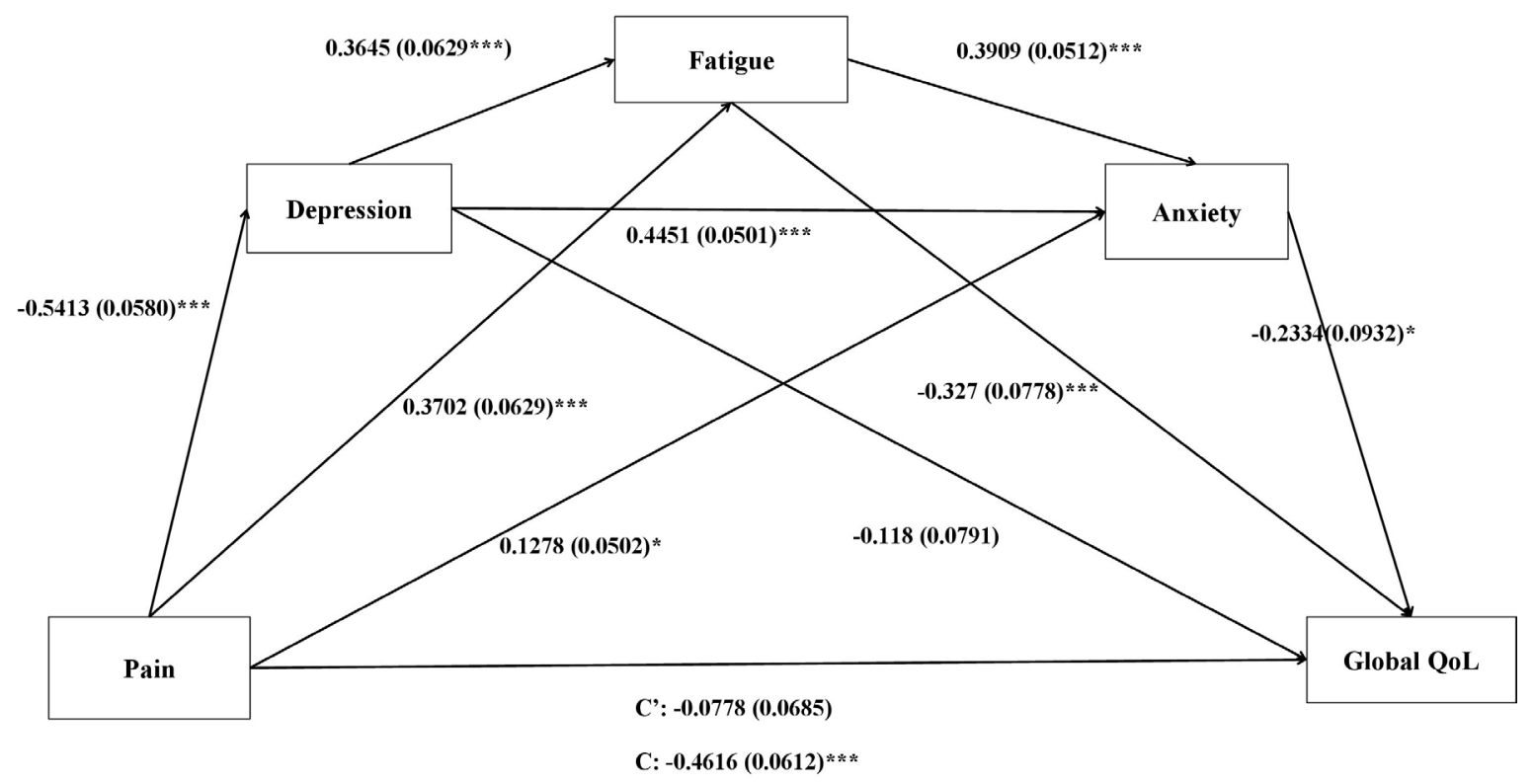

Figure 3 Serial mediation model 6 linking pain and quality of life $(Q \circ L)(n=212)$. Standardised effects are presented outside the parentheses with bootstrapped SEs in the parentheses. C'=direct effect of pain to HRQoL; C=total effect of pain to HRQoL; total indirect effect=-0.384, 95\% biased corrected Cl:-0.51 to -0.284 ; ratio of indirect to total effect: $0.832,95 \% \mathrm{Cl} 0.58$ to 1.17; model is controlled for cancer Diagnosis. Global fit indices: $\chi^{2}=10.27(3), p=0.069, C F I=0.938, R M S E A=0.10(0.04$, 0.18). ${ }^{*} \mathrm{P}<0.05,{ }^{* * *} \mathrm{P}<0.001$. CFI, Comparative Fit Index; HRQoL, health-related quality of life; RMSEA, root mean square error of approximation. 
worst the pain was the more it would contribute to depression, higher depression resulted to higher fatigue, higher fatigue led to higher levels of anxiety which in turn contributed to lower HRQoL.

\section{DISCUSSION}

The aim of this study was to explore the co-occurrence and inter-relations between symptoms that were reported by two groups of patients with cancer and HRQoL. With previous findings showing that these symptoms can be present in different clusters in these groups of patients, ${ }^{30}{ }^{31}$ we specifically aimed to demonstrate that pain, depression, fatigue, nausea, vomiting and retching and anxiety can form a common symptom cluster over the course of breast cancer and prostate cancer treatment. The findings showed that with the exception of nausea, vomiting and retching, the symptoms of pain, fatigue, anxiety and depression formed a common symptom cluster in patients with breast and prostate cancer. The findings also showed that HRQoL is negatively associated with pain, fatigue, anxiety and depression. This suggests that with these two groups of patients the symptoms consisting of the cluster need to be addressed collectively emphasising on pain management and focusing on depression, anxiety and fatigue. This provides a more comprehensive symptom management to the patient with also a positive effect on the HRQoL.

Parallel, serial and moderate mediation analyses yielded interesting results in relation to symptom correlations. Parallel mediation analysis showed that the three mediators (fatigue, anxiety and depression) fully mediate the relationship between pain and HRQoL. However, while anxiety and fatigue were found to significantly contribute to the overall IE, depression did not mediate the relationship between pain and HRQoL. The results demonstrated the mediating effect of these symptoms on pain and HRQoL, however, at the same time the results supported the notion that 'pain' needs to be targeted first. Although the data used for the analysis cannot support a definite model as these data were not longitudinal, however, it does suggest that researchers and clinicians should consider these alternative correlations between symptoms and HRQoL. For example, cancer diagnosis significantly moderated the mediational effect of fatigue but not anxiety. Therefore, for patients with prostate cancer conditional IE is minimal, but for patients with breast cancer, the effect is stronger and statistically significant. With the existence of many complex variable correlations being recorded in the literature in isolated symptom studies, these new findings suggest that conflicts in preceding studies may be resolved by the increased understanding of variable interaction in symptom clustering.

The exact underlying mechanisms by which symptoms correlate with each other are not to this date fully understood and this is an area that could contribute in the comprehensive management of symptom clusters through the development of effective multimodal interventions for patients with breast and prostate cancer for use in the active treatment phase. A variety of non-pharmacological interventions have been proposed for treating pain, fatigue, depression and anxiety including exercise, psychosocial, cognitive behavioural and nutritional. ${ }^{32-34}$ However, most of these recommendations are proposed for use in single symptom management, perhaps with the exception of GI and PMR that its effectiveness was also tested in symptom cluster. $^{29}$ Further research is needed towards examining whether multimodal interventions can reduce pain and, in turn, reduce depression, fatigue and anxiety.

Clinical decision-making in symptom management is traditionally driven by the type of the symptom rather than its impact on the patient or its correlations to other symptoms that might simultaneously be present. Preceding studies ${ }^{35}$ consistently showed that emphasis is given on pain management rather than management of other symptoms such as fatigue or anxiety within a clinical situation where a symptom cluster manifests. The main problem with these patterns in symptom management is that despite the knowledge that symptoms coexist; these are prioritised according to perceived importance. However, the correlation between the symptoms is purposively not taken into consideration including the likelihood that by treating, for example, fatigue in patients with breast cancer, the results of any pain management intervention might be fortified as well as the positive impact on HRQoL. On the same example, treating fatigue in patients with prostate cancer will most likely have no accumulating effect on the patient's responsiveness to pain management interventions. This example highlights that within the clinical setting such clinical decision-making has also an impact on the cost-effectiveness of the symptom management interventions, as resources can be purposively allocated to other symptoms or variables that mediate pain and HRQoL to maximise clinical management.

The study has some limitations. The main limitation is the small sample size; however, several statistically significant paths emerged and within-symptom paths were replicated across both study samples (ie, cancer type groups). The unavailability of longitudinal data limits the support of a definite model, which would demonstrate if the correlations between symptoms found in this study are stable over time. However, the fact that the patients in the study were all in the active treatment phase strengthens the generalisability of the results to patients with prostate and breast cancer during this phase.

The study demonstrated that pain, depression, fatigue, and anxiety tend to co-occur during the treatment phase, thus providing further evidence for this symptom cluster in two distinct samples of patients with breast and prostate cancer. The study also showed the direct and IEs of this symptom cluster on the person's HRQoL. Parallel mediation analysis showed that the three mediators (fatigue, anxiety and depression) fully mediate the relationship between pain and HRQoL. Similarly, in a serial causal order, the three mediators fully mediate the relationship between pain and HRQoL. The moderated mediation analysis showed that diagnosis significantly moderated the mediational effect of fatigue but did not moderate mediational effect of anxiety. There are explicit clinical implications of the study's findings 
that include assessment, prevention and intervention for anxiety, depression, fatigue and pain related to breast and prostate cancer treatment. This work provides preliminary evidence that targeting fatigue, anxiety and depression may have a meaningful effect on pain as a related symptom and potentially have a positive impact on HRQoL of patients with breast and prostate cancer.

Contributors The authors of this paper have directly participated in all the stages of its preparation. Authorship statement: AC conceived and designed the study, analysed, interpreted the data and approved the final manuscript. MG prepared all the draft versions of the manuscript. MG and LP recruited the participants, interpreted the data and edited and approved the final version of the manuscript. AC, MG and EB collected the data. LP performed the statistical analysis and interpretation of the data. $A C, M G$ and $L P$ interpreted the data, edited and approved the final version of the manuscript. AC and LP were responsible for the financial support.

Funding This work was supported by the Cyprus University of Technology. Improvast provided support in the form of salaries for author LP. Publication was supported through the Open Access Author's Fund of the Cyprus University of Technology.

Competing interests None declared.

Patient consent Obtained.

Ethics approval The study was compliant to the Declaration of Helsinki and the protocol was approved by the Cyprus National BioEthics Committee (ID CNBC/ $\mathrm{EP} / 2010 / 06)$.

Provenance and peer review Not commissioned; externally peer reviewed.

Data sharing statement Data are available from the Cyprus University of Technology given that the Cyprus National BioEthics Committee will grant access to data for researchers who meet the criteria for access to confidential data. There are no unpublished data from this study. Data requests can be sent to the corresponding author at andreas.charalambous@cut.ac.cy.

Open access This is an open access article distributed in accordance with the Creative Commons Attribution Non Commercial (CC BY-NC 4.0) license, which permits others to distribute, remix, adapt, build upon this work non-commercially, and license their derivative works on different terms, provided the original work is properly cited, appropriate credit is given, any changes made indicated, and the use is non-commercial. See: http://creativecommons.org/licenses/by-nc/4.0/.

\section{REFERENCES}

1. Dong ST, Butow PN, Costa DS, et al. Symptom clusters in patients with advanced cancer: a systematic review of observational studies. $J$ Pain Symptom Manage 2014;48:411-50.

2. Kim HJ, McGuire DB, Tulman L, et al. Symptom clusters: concept analysis and clinical implications for cancer nursing. Cancer Nurs 2005;28:270-82.

3. Miaskowski C, Dodd M, Lee K. Symptom clusters: the new frontier in symptom management research. J Natl Cancer Inst Monogr 2004:17-21.

4. Dodd MJ, Miaskowski C, Paul SM. Symptom clusters and their effect on the functional status of patients with cancer. Oncol Nurs Forum 2001;28:465-70.

5. Dodd M, Janson S, Facione N, et al. Advancing the science of symptom management. J Adv Nurs 2001;33:668-76.

6. Dodd MJ, Miaskowski C. Lee KA: Occurrence of symptom clusters. J Natl Cancer Inst Monogr:2004:76-78.

7. Dong ST, Costa DS, Butow PN, et al. Symptom Clusters in Advanced Cancer Patients: An Empirical Comparison of Statistical Methods and the Impact on Quality of Life. J Pain Symptom Manage 2016;51:88-98.

8. Preacher KJ, Rucker DD, Hayes AF. Addressing moderated mediation hypotheses: Theory, methods, and prescriptions. Multivariate Behav Res 2007;42:185-227.

9. Cheung WY, Le LW, Zimmermann C. Symptom clusters in patients with advanced cancers. Support Care Cancer 2009;17:1223-30.

10. Charalambous A, Giannakopoulou M, Bozas E, et al. Guided imagery and progressive muscle relaxation as a cluster of symptoms management intervention in patients receiving chemotherapy: A randomized control trial. PLoS One 2016;11:e0156911-8.
11. Jensen MP. The validity and reliability of pain measures in adults with cancer. J Pain 2003;4:2-21.

12. Chang VT, Hwang SS, Feuerman M, et al. Symptom and quality of life survey of medical oncology patients at a veterans affairs medical center: a role for symptom assessment. Cancer 2000;88:1175-83.

13. Okuyama T, Akechi T, Kugaya A, et al. Development and validation of the cancer fatigue scale: a brief, three-dimensional, self-rating scale for assessment of fatigue in cancer patients. J Pain Symptom Manage 2000;19:5-14.

14. Rhodes VA, McDaniel RW. The Index of Nausea, Vomiting, and Retching: a new format of the Index of Nausea and Vomiting. Oncol Nurs Forum 1999;26:889-93.

15. Lee J, Dibble SL, Pickett M, et al. Chemotherapy-induced nausea/ vomiting and functional status in women treated for breast cancer. Cancer Nurs 2005;28:249???255-55.

16. Zung WW. A rating instrument for anxiety disorders. Psychosomatics 1971;12:371-9.

17. Li M, Wang L. The Associations of Psychological Stress with Depressive and Anxiety Symptoms among Chinese Bladder and Renal Cancer Patients: The Mediating Role of Resilience. PLoS One 2016;11:e0154729.

18. Beck AT, Steer RA, Brown GK. Manual for the Beck Depression Inventory-II. San Antonio, TX: Psychological Corporation, 1996.

19. Hopko DR, Bell JL, Armento ME, et al. The phenomenology and screening of clinical depression in cancer patients. $J$ Psychosoc Oncol 2008;26:31-51.

20. Nho JH, Reul Kim S, Nam JH. Symptom clustering and quality of life in patients with ovarian cancer undergoing chemotherapy. Eur $J$ Oncol Nurs 2017:30:8-14.

21. Choi S, Ryu E. Effects of symptom clusters and depression on the quality of life in patients with advanced lung cancer. Eur $J$ Cancer Care 2018;27.

22. Fayers P, Bottomley A. on behalf of the EORTC Quality of Life Group and of the Quality of Life Unit: Quality of life research within the EORTC-the EORTC QLQ-C30. European Journal of Cancer 2002;38:S125-S133.

23. Tan ML, Idris DB, Teo LW, et al. Validation of EORTC QLQ-C30 and QLQ-BR23 questionnaires in the measurement of quality of life of breast cancer patients in Singapore. Asia Pac J Oncol Nurs 2014;1:22-32.

24. Sprangers MA, Groenvold M, Arraras Jl, et al. The European Organization for Research and Treatment of Cancer breast cancerspecific quality-of-life questionnaire module: first results from a three-country field study. J Clin Oncol 1996;14:2756-68.

25. van Andel G, Bottomley A, Fosså SD, et al. An international field study of the EORTC QLQ-PR25: a questionnaire for assessing the health-related quality of life of patients with prostate cancer. Eur $J$ Cancer 2008;44:2418-24.

26. Preacher KJ, Hayes AF. SPSS and SAS procedures for estimating indirect effects in simple mediation models. Behav Res Methods Instrum Comput 2004;36:717-31.

27. Hayes AF: Introduction to Mediation, Moderation, and Conditional Process Analysis : A Regressio n-Based Approach. New York, The Guilford Press 2013.

28. Rosseel Y. lavaan : An $R$ Package for Structural Equation Modeling. $J$ Stat Softw 2012;48:1-36.

29. Charalambous A, Giannakopoulou M, Bozas E, et al. A Randomized Controlled Trial for the Effectiveness of Progressive Muscle Relaxation and Guided Imagery as Anxiety Reducing Interventions in Breast and Prostate Cancer Patients Undergoing Chemotherapy. Evid Based Complement Alternat Med 2015;2015:1-10.

30. So WK, Marsh G, Ling WM, et al. The symptom cluster of fatigue, pain, anxiety, and depression and the effect on the quality of life of women receiving treatment for breast cancer: a multicenter study. Oncol Nurs Forum 2009;36:E205-14.

31. Dirksen SR, Belyea MJ, Wong W, et al. TRansitions in symptom cluster subgroups among men undergoing prostate cancer radiation therapy. Cancer Nurs 2016;39:3-11.

32. Wengström Y, Bolam KA, Mijwel S, et al. Optitrain: a randomised controlled exercise trial for women with breast cancer undergoing chemotherapy. BMC Cancer 2017; 17:100.

33. Finnegan-John J, Molassiotis A, Richardson A, et al. A systematic review of complementary and alternative medicine interventions for the management of cancer-related fatigue. Integr Cancer Ther 2013:12:276-90.

34. Santos Salas A, Fuentes Contreras J, Armijo-Olivo S, et al. Nonpharmacological cancer pain interventions in populations with social disparities: a systematic review and meta-analysis. Support Care Cancer 2016;24:985-1000.

35. Wilkie DJ, Ezenwa MO. Pain and symptom management in palliative care and at end of life. Nurs Outlook 2012;60:357-64. 